\title{
On the existence of solutions for a pointwise defined multi-singular integro-differential equation with integral boundary condition
}

\author{
Mandana Talaee ${ }^{1}$, Mehdi Shabibi² ${ }^{2}$ Alireza Gilani ${ }^{1}$ and Shahram Rezapour ${ }^{3,4^{*}}$
}

\author{
*Correspondence: \\ rezapourshahram@yahoo.ca \\ ${ }^{3}$ Department of Mathematics, \\ Azarbaijan Shahid Madani \\ University, Tabriz, Iran \\ ${ }^{4}$ Department of Medical Research, \\ China Medical University Hospital, \\ China Medical University, Taichung, \\ Taiwan \\ Full list of author information is \\ available at the end of the article
}

\begin{abstract}
It is important that we increase our ability for studying of complicate fractional integro-differential equation. In this paper, we investigates the existence of solutions for a pointwise defined multi-singular fractional differential equation under some integral boundary conditions. We provide an example to illustrate our main result.
\end{abstract}

MSC: Primary 34A08; secondary 34A60

Keywords: Multi-singularity; Pointwise defined fractional equation; The Caputo derivation

\section{Preliminaries}

We know that many researchers are working on fractional differential equarions from different point of view (see, for example, ([1-12] and [13]). In 2015, a new fractional derivative introduced entitled Caputo-Fabrizio and some researchers tried to obtain new techniques for studying of distinct integro-differential equations via the new derivation (see, for example, [14-19]) and new fractional models and optimal controls of different phenomena with the non-singular derivative operator (see, for example, [20-24] and [25]). Also, there has been published a lot of work about physical studies on fractional calculus and new aspects of fractional different models with Mittag-Leffler law (see, for example, [26-29] and [30]).

Most researchers like to obtain numerical solutions of fractional differential equations specially singular ones (see foe example, $[24,25]$ and [31]). It is natural that most softwares are not able to calculate solutions of most singular differential equations now while nowadays we can prove that most complicate problems such pointwise defined multi-singular fractional differential equations under some integral boundary conditions have solutions. But finding numerical solutions is not possible yet and this weakliness relates to the structures of the software.

In 2015, Liu and Wong investigated the fractional problem ${ }^{c} D^{\alpha} x(t)=f\left(t, x(t), D^{\beta} x(t)\right)$ with boundary conditions $x(0)+x^{\prime}(0)=y(x), \int_{0}^{1} x(t) d t=m$ and $x^{\prime \prime}(0)=x^{(3)}(0)=\cdots=$ $x^{(n-1)}(0)=0$, where $0<t<1, m$ is a real number, $n \geq 2, \alpha \in(n-1, n), 0<\beta<1, D^{\alpha}$ and

(c) The Author(s) 2020. This article is licensed under a Creative Commons Attribution 4.0 International License, which permits use sharing, adaptation, distribution and reproduction in any medium or format, as long as you give appropriate credit to the original author(s) and the source, provide a link to the Creative Commons licence, and indicate if changes were made. The images or other third party material in this article are included in the article's Creative Commons licence, unless indicated otherwise in a credit line to the material. If material is not included in the article's Creative Commons licence and your intended use is not permitted by statutory regulation or exceeds the permitted use, you will need to obtain permission directly from the copyright holder. To view a copy of this licence, visit http://creativecommons.org/licenses/by/4.0/. 
$D^{\beta}$ are the Caputo fractional derivatives, $y \in C_{\mathbb{R}}([0,1])$ and $f:(0,1] \times \mathbb{R} \times \mathbb{R} \rightarrow \mathbb{R}$ is continuous with $f(t, x, y)$ may be singular at $t=0$ [32]. In 2016, Shabibi et al. introduced a new type of fractional differential equations entitled pointwise defined integro-differential problems [33]. Recall that $D^{\alpha} x(t)+f(t)=0$ is a pointwise defined equation on [0,1] if there exists a set $E \subset[0,1]$ such that the measure of $E^{c}$ is zero and the equation holds on $E$ [33]. In 2018, Baleanu et al. reviewed the existence of solutions for the pointwise defined three steps crisis integro-differential equation

$$
D^{\alpha} x(t)+f\left(t, x(t), x^{\prime}(t), D^{\beta} x(t), \int_{0}^{t} h(\xi) x(\xi) d \xi, \phi(x(t))\right)=0
$$

with boundary conditions $x(1)=x(0)=x^{\prime \prime}(0)=x^{n}(0)=0$, where $\alpha \geq 2, \lambda, \mu, \beta \in(0,1), \phi$ : $X \rightarrow X$ is a mapping such that $\|\phi(x)-\phi(y)\| \leq \theta_{0}\|x-y\|+\theta_{1}\left\|x^{\prime}-y^{\prime}\right\|$ for some non-negative real numbers $\theta_{0}$ and $\theta_{1} \in[0, \infty)$ and all $x, y \in X, D^{\alpha}$ is the Caputo fractional derivative of order $\alpha, f\left(t, x_{1}(t), \ldots, x_{5}(t)\right)=f_{1}\left(t, x_{1}(t), \ldots, x_{5}(t)\right)$ for all $t \in[0, \lambda), f\left(t, x_{1}(t), \ldots, x_{5}(t)\right)=$ $f_{2}\left(t, x_{1}(t), \ldots, x_{5}(t)\right)$ for all $t \in[\lambda, \mu]$ and $f\left(t, x_{1}(t), \ldots, x_{5}(t)\right)=f\left(t, x_{1}(t), \ldots, x_{5}(t)\right)$ for all $t \in(\mu, 1], f_{1}(t, \cdot, \cdot, \cdot, \cdot)$ and $f_{3}(t, \cdot, \cdot, \cdot, \cdot)$ are continuous on $[0, \lambda)$ and $(\mu, 1]$ and $f_{2}(t, \cdot, \cdot, \cdot, \cdot)$ is multi-singular [34]. In 2019, Chergui et al. reviewed the existence and uniqueness of solution for the nonlinear fractional boundary value problem $D^{q} x(t)=f\left(t, x(t), D^{r} x(t)\right)$ with non-separated type integral boundary conditions $x(0)-\lambda_{1} x(T)=\mu_{1} \int_{0}^{T} g(s, x(s)) d s$ and $x^{\prime}(0)-\lambda_{2} x^{\prime}(T)=\mu_{2} \int_{0}^{T} h(s, x(s)) d s$, where $t \in[0, T], 1<q \leq 2,0<r \leq 1, D^{q}$ is the Caputo fractional derivative of order $q, f \in C_{\mathbb{R}}([0, T] \times \mathbb{R} \times \mathbb{R}), g, h:[0, T] \times \mathbb{R} \rightarrow \mathbb{R}$ are given continuous functions and $\lambda_{1}, \lambda_{2}, \mu_{1}, \mu_{2} \in \mathbb{R}$ with $\lambda_{1} \neq 1$ and $\lambda_{2} \neq 1$ [35].

Motivated by the work, we investigate the existence of solutions for the nonlinear fractional differential pointwise defined problem

$$
D^{\alpha} x(t)=f\left(t, x(t), x^{\prime}(t), D^{\beta} x(t), \int_{0}^{t} g(\xi) x(\xi) d \xi\right)
$$

with boundary conditions $x(\mu)=\int_{0}^{1} g(z) x(z) d z$ and $x(0)=x^{(j)}(0)=0$, for $2 \leq j \leq n-1$, where $\alpha \geq 2, n=[\alpha]+1, \mu, \beta \in(0,1), g, h:[0,1] \rightarrow \mathbb{R}$ are two maps such that $g, h \in L^{1}[0,1]$ and $f \in L^{1}$ is singular at some points $[0,1]$. Here, $\|\cdot\|_{1}$ denotes the norm of $L^{1}[0,1]$. We consider the sup norm $\|\cdot\|$ for $Y=C[0,1]$ and $\|x\|_{*}=\max \left\{\|x\|,\left\|x^{\prime}\right\|\right\}$ for $C^{1}[0,1]$. The Riemann-Liouville integral of order $p$ with the lower limit $a \geq 0$ for a function $f:(a, \infty) \rightarrow$ $\mathbb{R}$ is defined by $I_{a^{+}}^{p} f(t)=\frac{1}{\Gamma(p)} \int_{a}^{t}(t-s)^{p-1} f(s) d s$ provided that the right-hand side is pointwise defined on $(a, \infty)$. we denote $I^{p} f(t)$ for $I_{0^{+}}^{p} f(t)$ [36]. The Caputo fractional derivative of order $\alpha>0$ of a function $f:(a, \infty) \rightarrow \mathbb{R}$ is defined by ${ }^{c} D^{\alpha} f(t)=\frac{1}{\Gamma(n-\alpha)} \int_{0}^{t} \frac{f^{n}(s)}{(t-s)^{\alpha+1-n}} d s$, where $n=[\alpha]+1[36]$. We need the following results.

Lemma 1 ([37]) Let $0<n-1 \leq \alpha<n$ and $x \in C(0,1)$. Then there exist real constants $c_{0}, \ldots, c_{n-1}$ such that $D^{\alpha} x(t)=x(t)+\sum_{i=0}^{n-1} c_{i} t^{i}$.

Lemma 2 ([38]) Let $X$ be a Banach space, $C$ a closed and convex of $X, \Omega$ a relatively open subset of $C$ with $0 \in \Omega$ and $F: \Omega \rightarrow C$ a continuous and compact map. Then either $F$ has a fixed point in $\bar{\Omega}$ or there exist $y \in \partial \Omega$ and $\lambda \in(0,1)$ such that $y=\lambda F y$. 


\section{Main results}

Lemma 3 Let $\alpha \geq 2, n=[\alpha]+1, \mu, \beta \in(0,1), f, h:[0,1] \rightarrow \mathbb{R}$ such that $f, h \in L^{1}[0,1]$ and $\mu \neq \int_{0}^{1} z h(z) d z$. A map $x$ is a solution for the pointwise defined equation $D^{\alpha} x(t)=f(t)$ with boundary conditions $x(\mu)=\int_{0}^{1} h(z) x(z) d z$ and $x(0)=x^{(j)}(0)=0(2 \leq j \leq n)$ if and only if $x(t)=\int_{0}^{1} G(t, s) f(s) d s$, where

$$
G(t, s)= \begin{cases}\frac{(t-s)^{\alpha-1}}{\Gamma(\alpha)}+\frac{t\left[(\mu-s)^{\alpha}-H_{\alpha}(s)\right]}{A_{\mu} \Gamma(\alpha)}, & 0 \leq s \leq t \leq 1, \mu \geq s, \\ \frac{(t-s)^{\alpha-1}}{\Gamma(\alpha)}-\frac{t H_{\alpha}(s)}{A_{\mu} \Gamma(\alpha)}, & 0 \leq \mu \leq s \leq t \leq 1, \\ \frac{t\left((\mu-s)^{\alpha}-H_{\alpha}(s)\right]}{A_{\mu} \Gamma(\alpha)}, & 0 \leq t \leq s \leq \mu \leq 1, \\ -\frac{t H_{\alpha}(s)}{A_{\mu} \Gamma(\alpha)}, & 0 \leq t \leq s \leq 1, \mu \leq s,\end{cases}
$$

$H_{\alpha}(t)=\int_{t}^{1}(z-t)^{\alpha-1} h(z) d z$ and $A_{\mu}=\int_{0}^{1}(z h(z)-\mu) d z$.

Proof By following the related proof in [34], we concluded that Lemma 1 is valid on $L^{1}[0,1]$. Now let $x(t)$ be a solution for the problem. By using Lemma 1 and $x^{(j)}(0)=0$ for $j \geq 2$, we get $x(t)=\frac{1}{\Gamma(\alpha)} \int_{0}^{t}(t-s)^{\alpha-1} f(s) d s+c_{0}+c_{1} t$. Since $x(0)=0, c_{0}=0$ and so $x(t)=\frac{1}{\Gamma(\alpha)} \int_{0}^{t}(t-s)^{\alpha-1} f(s) d s+c_{1} t$. Hence,

$$
x(\mu)=\frac{1}{\Gamma(\alpha)} \int_{0}^{\mu}(\mu-s)^{\alpha-1} f(s) d s+c_{1} \mu
$$

and $h(z) x(z)=\frac{1}{\Gamma(\alpha)} h(z) \int_{0}^{z}(z-s)^{\alpha-1} f(s) d s+c_{1} z h(z)$ for all $z \in[0,1]$. Thus,

$$
\int_{0}^{1} h(z) x(z) d z=\frac{1}{\Gamma(\alpha)} \int_{0}^{1} \int_{0}^{z}(z-s)^{\alpha-1} h(z) f(s) d s d z+c_{1} \int_{0}^{1} z h(z) d z
$$

and so $\int_{0}^{1} h(z) x(z) d z=\frac{1}{\Gamma(\alpha)} \int_{0}^{1} \int_{s}^{1}(z-s)^{\alpha-1} h(z) f(s) d z d s+c_{1} \int_{0}^{1} z h(z) d z$. Put

$$
H_{\alpha}(t)=\int_{t}^{1}(z-t)^{\alpha-1} h(z) d z
$$

Then we have $\int_{0}^{1} h(z) x(z) d z=\frac{1}{\Gamma(\alpha)} \int_{0}^{1} H_{\alpha}(s) f(s) d s+c_{1} \int_{0}^{1} z h(z) d z$. By using the assumption $x(\mu)=\int_{0}^{1} z h(z) d z$ and (2), we obtain

$$
\frac{1}{\Gamma(\alpha)} \int_{0}^{\mu}(\mu-s)^{\alpha-1} f(s) d s+c_{1} \mu=\frac{1}{\Gamma(\alpha)} \int_{0}^{1} H_{\alpha}(s) f(s) d s+c_{1} \int_{0}^{1} z h(z) d z .
$$

Hence, $c_{1}\left(\int_{0}^{1} z h(z) d z-\mu\right)=\frac{1}{\Gamma(\alpha)} \int_{0}^{\mu}(\mu-s)^{\alpha-1} f(s) d s-\frac{1}{\Gamma(\alpha)} \int_{0}^{1} H_{\alpha}(s) f(s) d s$ and so

$$
c_{1}=\frac{1}{A_{\mu} \Gamma(\alpha)} \int_{0}^{\mu}(\mu-s)^{\alpha-1} f(s) d s-\frac{1}{A_{\mu} \Gamma(\alpha)} \int_{0}^{1} H_{\alpha}(s) f(s) d s,
$$

where $A_{\mu}=\int_{0}^{1}(z h(z)-\mu) d z$. Thus,

$$
x(t)=\frac{1}{\Gamma(\alpha)} \int_{0}^{t}(t-s)^{\alpha-1} f(s) d s+\frac{t}{A_{\mu} \Gamma(\alpha)} \int_{0}^{\mu}(\mu-s)^{\alpha-1} f(s) d s
$$




$$
-\frac{t}{A_{\mu} \Gamma(\alpha)} \int_{0}^{1} H_{\alpha}(s) f(s) d s
$$

If $\mu \leq t$, then

$$
\begin{aligned}
x(t)= & \frac{1}{\Gamma(\alpha)}\left(\int_{0}^{\mu}+\int_{\mu}^{t}\right)(t-s)^{\alpha-1} f(s) d s+\frac{t}{A_{\mu} \Gamma(\alpha)} \int_{0}^{\mu}(\mu-s)^{\alpha-1} f(s) d s \\
& -\frac{t}{A_{\mu} \Gamma(\alpha)}\left(\int_{0}^{\mu}+\int_{\mu}^{t}+\int_{t}^{1}\right) H_{\alpha}(s) f(s) d s,
\end{aligned}
$$

and for $t \leq \mu$ we have

$$
\begin{aligned}
x(t)= & \frac{1}{\Gamma(\alpha)} \int_{0}^{t}(t-s)^{\alpha-1} f(s) d s+\frac{t}{A_{\mu} \Gamma(\alpha)}\left(\int_{0}^{t}+\int_{t}^{\mu}\right)(\mu-s)^{\alpha-1} f(s) d s \\
& -\frac{t}{A_{\mu} \Gamma(\alpha)}\left(\int_{0}^{t}+\int_{t}^{\mu}+\int_{\mu}^{1}\right) H_{\alpha}(s) f(s) d s .
\end{aligned}
$$

Hence $x(t)$ is given by $x(t)=\int_{0}^{1} G(t, s) f(s) d s$, where

$$
G(t, s)= \begin{cases}\frac{(t-s)^{\alpha-1}}{\Gamma(\alpha)}+\frac{t\left[(\mu-s)^{\alpha}-H_{\alpha}(s)\right]}{A_{\mu} \Gamma(\alpha)}, & 0 \leq s \leq t \leq 1, \mu \geq s, \\ \frac{(t-s)^{\alpha-1}}{\Gamma(\alpha)}-\frac{t H_{\alpha}(s)}{A_{\mu} \Gamma(\alpha)}, & 0 \leq \mu \leq s \leq t \leq 1, \\ \frac{t\left[(\mu-s)^{\alpha}-H_{\alpha}(s)\right]}{A_{\mu} \Gamma(\alpha)}, & 0 \leq t \leq s \leq \mu \leq 1, \\ -\frac{t H_{\alpha}(s)}{A_{\mu} \Gamma(\alpha)}, & 0 \leq t \leq s \leq 1, \mu \leq s .\end{cases}
$$

The converse part could be obtained easily by some straightforward calculations.

Note that

$$
\frac{\partial G}{\partial t}(t, s)= \begin{cases}\frac{(t-s)^{\alpha-2}}{\Gamma(\alpha-1)}+\frac{(\mu-s)^{\alpha}-H_{\alpha}(s)}{A_{\mu} \Gamma(\alpha)}, & 0 \leq s \leq t \leq 1, \mu \geq s, \\ \frac{(t-s)^{\alpha-2}}{\Gamma(\alpha-1)}-\frac{H_{\alpha}(s)}{A_{\mu} \Gamma(\alpha)}, & 0 \leq \mu \leq s \leq t \leq 1, \\ \frac{(\mu-s)^{\alpha}-H_{\alpha}(s)}{A_{\mu} \Gamma(\alpha)}, & 0 \leq t \leq s \leq \mu \leq 1, \\ -\frac{H_{\alpha}(s)}{A_{\mu} \Gamma(\alpha)}, & 0 \leq t \leq s \leq 1, \mu \leq s\end{cases}
$$

and so $G$ and $\frac{\partial}{\partial t} G$ are continuous with respect to $t$. Assume that $f \in L^{1}([0,1] \times \mathbb{R} \times \mathbb{R} \times$ $\mathbb{R} \times \mathbb{R})$ is a map such that $f$ is singular at some points of $[0,1]$. Define the map $F: X \rightarrow X$ by

$$
\begin{aligned}
F_{x}(t)= & \int_{0}^{1} G(t, s) f\left(s, x(s), x^{\prime}(s), D^{\beta} x(s), \int_{0}^{s} g(\xi) x(\xi) d \xi\right) d s \\
= & \frac{1}{\Gamma(\alpha)} \int_{0}^{t}(t-s)^{\alpha-1} f\left(s, x(s), x^{\prime}(s), D^{\beta} x(s), \int_{0}^{s} g(\xi) x(\xi) d \xi\right) d s \\
& +\frac{t}{A_{\mu} \Gamma(\alpha)} \int_{0}^{\mu}(\mu-s)^{\alpha-1} f\left(s, x(s), x^{\prime}(s), D^{\beta} x(s), \int_{0}^{s} g(\xi) x(\xi) d \xi\right) d s \\
& -\frac{t}{A_{\mu} \Gamma(\alpha)} \int_{0}^{1} H_{\alpha}(s) f\left(s, x(s), x^{\prime}(s), D^{\beta} x(s), \int_{0}^{s} g(\xi) x(\xi) d \xi\right) d s
\end{aligned}
$$


for all $t \in[0,1]$, where $g:[0,1] \rightarrow \mathbb{R}$ belongs to $L^{1}[0,1]$. Note that, $\left|D^{\beta} x\right| \leq \frac{\left\|x^{\prime}\right\|}{\Gamma(2-\beta)}$ and $\left|\int_{0}^{s} g(\xi) x(\xi) d \xi\right| \leq m\|x\|$, where $m=\int_{0}^{1}|g(\xi)| d \xi$. Let $t \in[0,1]$. Then we have

$$
\begin{aligned}
F_{x}^{\prime}(t)= & \int_{0}^{1} \frac{\partial G}{\partial t}(t, s) f\left(s, x(s), x^{\prime}(s), D^{\beta} x(s), \int_{0}^{s} g(\xi) x(\xi) d \xi\right) d s \\
= & \frac{1}{\Gamma(\alpha-1)} \int_{0}^{t}(t-s)^{\alpha-2} f\left(s, x(s), x^{\prime}(s), D^{\beta} x(s), \int_{0}^{s} g(\xi) x(\xi) d \xi\right) d s \\
& +\frac{1}{A_{\mu} \Gamma(\alpha)} \int_{0}^{\mu}(\mu-s)^{\alpha-1} f\left(s, x(s), x^{\prime}(s), D^{\beta} x(s), \int_{0}^{s} g(\xi) x(\xi) d \xi\right) d s \\
& -\frac{1}{A_{\mu} \Gamma(\alpha)} \int_{0}^{1} H_{\alpha}(s) f\left(s, x(s), x^{\prime}(s), D^{\beta} x(s), \int_{0}^{s} g(\xi) x(\xi) d \xi\right) d s .
\end{aligned}
$$

Note that $x_{0} \in X$ is a solution for the singular pointwise defined equation (1) if and only if $x_{0}$ is a fixed point of $F$.

Theorem 4 Let $\alpha \geq 2, n=[\alpha]+1, \mu, \beta \in(0,1), g, h:[0,1] \rightarrow \mathbb{R}$ are mappings such that $g, h \in L^{1}[0,1]$ with $\mu \neq \int_{0}^{1} z h(z) d z, \Delta:=\max \left\{1, m, \frac{1}{\Gamma(2-\beta)}\right\}$ and $m:=\|g\|_{1}$. Assume that $f:$ $[0,1] \times X^{4} \rightarrow \mathbb{R}$ is a mapping such that $f$ is singular on some point of $[0,1]$ and for all $x_{1}, \ldots, x_{4}, y_{1}, \ldots, y_{4} \in X$ and for almost all $t \in[0,1]$ we have

$$
\left|f\left(t, x_{1}, x_{2}, \ldots, x_{4}\right)-f\left(t, y_{1}, y_{2}, \ldots, y_{4}\right)\right| \leq \sum_{i=1}^{4} a_{i}(t) \Lambda_{i}\left(\left|x_{i}-y_{i}\right|\right)
$$

where $a_{i}:[0,1] \rightarrow \mathbb{R}^{+}, \hat{a}_{i} \in L^{1}[0,1], \hat{a}_{i}(s)=(1-s)^{\alpha-2} a_{i}(s), \Lambda_{i}: \mathbb{R}^{+} \rightarrow \mathbb{R}^{+}$is a nondecreasing mapping with respect to all their components such that $\lim _{z \rightarrow 0^{+}} \frac{\Lambda_{i}(z)}{g_{i}(z)}=q_{i}$ for some $q_{i} \geq 0$. Suppose that $g_{1}, \ldots, g_{4}: \mathbb{R} \rightarrow \mathbb{R}^{+}$are some functions such that $\lim _{z \rightarrow 0^{+}} g_{i}(z)=0$ for $1 \leq i \leq 4$. Also, assume that for almost all $t \in[0,1]$ and $x_{1}, \ldots, x_{4} \in X$ we have

$$
\left|f\left(t, x_{1}, x_{2}, \ldots, x_{4}\right)\right| \leq \sum_{i=1}^{k_{0}} \theta_{i}(t) M_{i}\left(x_{1}, \ldots, x_{4}\right)+N\left(x_{1}, \ldots, x_{4}\right),
$$

where $k_{0} \in \mathbb{N}, \theta_{i}:[0,1] \rightarrow \mathbb{R}^{+}, \hat{\theta}_{i} \in L^{1}[0,1], M_{i}, N: \mathbb{R}^{4} \rightarrow[0, \infty)$ are nondecreasing mappings with respect to all their components, $\lim _{z \rightarrow \infty} \frac{M_{i}(z, \ldots, z)}{z}=m_{i} \in(0, \infty)$ and $\lim _{z \rightarrow \infty} N(z$, $\ldots, z)<\infty$.If $\left(\frac{1}{\Gamma(\alpha-1)}+\frac{1+\|h\|_{1}}{\left|A_{\mu}\right| \Gamma(\alpha)}\right) \sum_{i=1}^{k_{0}} m_{i}\left\|\hat{\theta}_{i}\right\|_{[0,1]} \in\left(0, \frac{1}{\Delta}\right)$, then the problem $(1)$ has a solution.

Proof We show that the map $F$ is continuous. For $x, y \in X$ and $t \in[0,1]$, we have

$$
\begin{aligned}
\mid F_{x}(t) & -F_{y}(t) \mid \\
\leq & \mid \int_{0}^{1} G(t, s) f\left(s, x(s), x^{\prime}(s), D^{\beta} x(s), \int_{0}^{s} g(\xi) x(\xi) d \xi\right) d s \\
& -\int_{0}^{1} G(t, s) f\left(s, y(s), y^{\prime}(s), D^{\beta} y(s), \int_{0}^{s} g(\xi) y(\xi) d \xi\right) \mid d s \\
\leq & \frac{1}{\Gamma(\alpha)} \int_{0}^{t}(t-s)^{\alpha-1} \mid f\left(s, x(s), x^{\prime}(s), D^{\beta} x(s), \int_{0}^{s} g(\xi) x(\xi) d \xi\right) \\
& -f\left(s, y(s), y^{\prime}(s), D^{\beta} y(s), \int_{0}^{s} g(\xi) y(\xi) d \xi\right) \mid d s
\end{aligned}
$$




$$
\begin{aligned}
& +\frac{t}{\left|A_{\mu}\right| \Gamma(\alpha)} \int_{0}^{\mu}(\mu-s)^{\alpha-1} \mid f\left(s, x(s), x^{\prime}(s), D^{\beta} x(s), \int_{0}^{s} g(\xi) x(\xi) d \xi\right) \\
& -f\left(s, y(s), y^{\prime}(s), D^{\beta} y(s), \int_{0}^{s} g(\xi) y(\xi) d \xi\right) \mid d s \\
& +\frac{t}{\left|A_{\mu}\right| \Gamma(\alpha)} \int_{0}^{1}\left|H_{\alpha}(s)\right| f\left(s, x(s), x^{\prime}(s), D^{\beta} x(s), \int_{0}^{s} g(\xi) x(\xi) d \xi\right) \\
& -f\left(s, y(s), y^{\prime}(s), D^{\beta} y(s), \int_{0}^{s} g(\xi) y(\xi) d \xi\right) \mid d s \\
& \leq \frac{1}{\Gamma(\alpha)} \int_{0}^{t}(t-s)^{\alpha-1}\left[a_{1}(s) \Lambda_{1}(|x(s)-y(s)|)+a_{2}(s) \Lambda_{2}\left(\left|x^{\prime}(s)-y^{\prime}(s)\right|\right)\right. \\
& \left.+a_{3}(s) \Lambda_{3}\left(\left|D^{\beta} x(s)-D^{\beta} y(s)\right|\right)+a_{4}(s) \Lambda_{4}\left(\left|\int_{0}^{s} g(\xi)(x(\xi)-y(\xi)) d \xi\right|\right)\right] d s \\
& +\frac{t}{\left|A_{\mu}\right| \Gamma(\alpha)} \int_{0}^{\mu}(\mu-s)^{\alpha-1}\left[a_{1}(s) \Lambda_{1}(|x(s)-y(s)|)+a_{2}(s) \Lambda_{2}\left(\left|x^{\prime}(s)-y^{\prime}(s)\right|\right)\right. \\
& \left.+a_{3}(s) \Lambda_{3}\left(\left|D^{\beta} x(s)-D^{\beta} y(s)\right|\right)+a_{4}(s) \Lambda_{4}\left(\left|\int_{0}^{s} g(\xi)(x(\xi)-y(\xi)) d \xi\right|\right)\right] d s \\
& +\frac{t}{\left|A_{\mu}\right| \Gamma(\alpha)} \int_{0}^{1}\left|H_{\alpha}(s)\right|\left[a_{1}(s) \Lambda_{1}(|x(s)-y(s)|)+a_{2}(s) \Lambda_{2}\left(\left|x^{\prime}(s)-y^{\prime}(s)\right|\right)\right. \\
& \left.+a_{3}(s) \Lambda_{3}\left(\left|D^{\beta} x(s)-D^{\beta} y(s)\right|\right)+a_{4}(s) \Lambda_{4}\left(\left|\int_{0}^{s} g(\xi)(x(\xi)-y(\xi)) d \xi\right|\right)\right] d s \\
& \leq \frac{1}{\Gamma(\alpha)} \int_{0}^{t}(t-s)^{\alpha-1}\left[a_{1}(s) \Lambda_{1}(\|x-y\|)+a_{2}(s) \Lambda_{2}\left(\left\|x^{\prime}-y^{\prime}\right\|\right)\right. \\
& \left.\left.+a_{3}(s) \Lambda_{3}\left(\frac{\left\|x^{\prime}-y^{\prime}\right\|}{\Gamma(2-\beta)}\right)+a_{4}(s) \Lambda_{4}(m\|x-y\|)\right)\right] d s \\
& +\frac{t}{\left|A_{\mu}\right| \Gamma(\alpha)} \int_{0}^{\mu}(\mu-s)^{\alpha-1}\left[a_{1}(s) \Lambda_{1}(\|x-y\|)+a_{2}(s) \Lambda_{2}\left(\left\|x^{\prime}-y^{\prime}\right\|\right)\right. \\
& \left.+a_{3}(s) \Lambda_{3}\left(\frac{\left\|x^{\prime}-y^{\prime}\right\|}{\Gamma(2-\beta)}\right)+a_{4}(s) \Lambda_{4}(m\|x-y\|)\right] d s \\
& +\frac{t}{\left|A_{\mu}\right| \Gamma(\alpha)} \int_{0}^{1}\left|H_{\alpha}(s)\right|\left[a_{1}(s) \Lambda_{1}(\|x-y\|)+a_{2}(s) \Lambda_{2}\left(\left\|x^{\prime}-y^{\prime}\right\|\right)\right. \\
& \left.+a_{3}(s) \Lambda_{3}\left(\frac{\left\|x^{\prime}-y^{\prime}\right\|}{\Gamma(2-\beta)}\right)+a_{4}(s) \Lambda_{4}(m\|x-y\|)\right] d s \\
& \leq \frac{1}{\Gamma(\alpha)} \int_{0}^{t}(t-s)^{\alpha-1}\left[a_{1}(s) \Lambda_{1}(\Delta\|x-y\|)+a_{2}(s) \Lambda_{2}\left(\Delta\left\|x^{\prime}-y^{\prime}\right\|\right)\right. \\
& \left.+a_{3}(s) \Lambda_{3}\left(\Delta\left\|x^{\prime}-y^{\prime}\right\|\right)+a_{4}(s) \Lambda_{4}(\Delta\|x-y\|)\right] d s \\
& +\frac{t}{\left|A_{\mu}\right| \Gamma(\alpha)} \int_{0}^{\mu}(\mu-s)^{\alpha-1}\left[a_{1}(s) \Lambda_{1}(\Delta\|x-y\|)+a_{2}(s) \Lambda_{2}\left(\Delta\left\|x^{\prime}-y^{\prime}\right\|\right)\right. \\
& \left.+a_{3}(s) \Lambda_{3}\left(\Delta\left\|x^{\prime}-y^{\prime}\right\|\right)+a_{4}(s) \Lambda_{4}(\Delta\|x-y\|)\right] d s \\
& +\frac{t}{\left|A_{\mu}\right| \Gamma(\alpha)} \int_{0}^{1}\left|H_{\alpha}(s)\right|\left[a_{1}(s) \Lambda_{1}(\Delta\|x-y\|)+a_{2}(s) \Lambda_{2}\left(\Delta\left\|x^{\prime}-y^{\prime}\right\|\right)\right. \\
& \left.+a_{3}(s) \Lambda_{3}\left(\Delta\left\|x^{\prime}-y^{\prime}\right\|\right)+a_{4}(s) \Lambda_{4}(\Delta\|x-y\|)\right] d s,
\end{aligned}
$$


where $\Delta:=\max \left\{1, m, \frac{1}{\Gamma(2-\beta)}\right\}$. Hence,

$$
\begin{aligned}
\mid F_{x}(t) & -F_{y}(t) \mid \\
\leq & \frac{1}{\Gamma(\alpha)} \int_{0}^{t}(t-s)^{\alpha-1}\left[a_{1}(s) \Lambda_{1}\left(\Delta\|x-y\|_{*}\right)+a_{2}(s) \Lambda_{2}\left(\Delta\|x-y\|_{*}\right)\right. \\
& \left.+a_{3}(s) \Lambda_{3}\left(\Delta\|x-y\|_{*}\right)+a_{4}(s) \Lambda_{4}\left(\Delta\|x-y\|_{*}\right)\right] d s \\
& +\frac{t}{\left|A_{\mu}\right| \Gamma(\alpha)} \int_{0}^{\mu}(\mu-s)^{\alpha-1}\left[a_{1}(s) \Lambda_{1}\left(\Delta\|x-y\|_{*}\right)+a_{2}(s) \Lambda_{2}\left(\Delta\|x-y\|_{*}\right)\right. \\
& \left.+a_{3}(s) \Lambda_{3}\left(\Delta\|x-y\|_{*}\right)+a_{4}(s) \Lambda_{4}\left(\Delta\|x-y\|_{*}\right)\right] d s \\
& +\frac{t}{\left|A_{\mu}\right| \Gamma(\alpha)} \int_{0}^{1}\left|H_{\alpha}(s)\right|\left[a_{1}(s) \Lambda_{1}\left(\Delta\|x-y\|_{*}\right)+a_{2}(s) \Lambda_{2}\left(\Delta\|x-y\|_{*}\right)\right. \\
& \left.+a_{3}(s) \Lambda_{3}\left(\Delta\|x-y\|_{*}\right)+a_{4}(s) \Lambda_{4}\left(\Delta\|x-y\|_{*}\right)\right] d s .
\end{aligned}
$$

Since $\lim _{z \rightarrow 0^{+}} g_{i}(z)=0$, for $\epsilon>0$ there exists $\delta_{g}>0$ such that $0<z<\delta_{g}$ indicates that $\left|g_{i}(z)\right|<$ $\epsilon$, for all $1 \leq i \leq 4$. On the other hand by using $\lim _{z \rightarrow 0^{+}} \frac{\Lambda_{i}(z)}{g_{i}(z)}=q_{i}$, for each $\epsilon>0$ there exists $\delta_{0}>0$ such that $\frac{\Lambda_{i}(z)}{g_{i}(z)}<q_{i}+\epsilon$ for $0<z<\delta_{0}$ and $1 \leq i \leq 4$. Thus,

$$
\Lambda_{i}(z)<\left(q_{i}+\epsilon\right) g_{i}(z)
$$

for $0<z<\delta_{0}$. Put $\delta=\min \left\{\delta_{0}, \delta_{g}, \epsilon\right\}$. Then we have

$$
\Lambda_{i}(z)<\left(q_{i}+\epsilon\right) \epsilon
$$

for $0<z<\delta$ and $1 \leq i \leq 4$. Let $\|x-y\|_{*}<\frac{\delta}{\Delta}$. By using (5) we have

$$
\Lambda_{i}\left(\Delta\|x-y\|_{*}\right)<\left(q_{i}+\epsilon\right) \epsilon
$$

for all $1 \leq i \leq 4$. Thus by using (4), for $\|x-y\|_{*}<\frac{\delta}{\Delta}$ we get

$$
\begin{aligned}
\mid F_{x}(t) & -F_{y}(t) \mid \\
\leq & \frac{1}{\Gamma(\alpha)} \int_{0}^{t}(t-s)^{\alpha-1}\left[a_{1}(s)\left(q_{1}+\epsilon\right) \epsilon+\cdots+a_{4}(s) \Lambda_{4}\left(q_{4}+\epsilon\right) \epsilon\right] d s \\
& +\frac{t}{\left|A_{\mu}\right| \Gamma(\alpha)} \int_{0}^{\mu}(\mu-s)^{\alpha-1}\left[a_{1}(s)\left(q_{1}+\epsilon\right) \epsilon+\cdots+a_{4}(s) \Lambda_{4}\left(q_{4}+\epsilon\right) \epsilon\right] d s \\
& +\frac{t}{\left|A_{\mu}\right| \Gamma(\alpha)} \int_{0}^{1}\left|H_{\alpha}(s)\right|\left[a_{1}(s)\left(q_{1}+\epsilon\right) \epsilon+\cdots+a_{4}(s) \Lambda_{4}\left(q_{4}+\epsilon\right) \epsilon\right] d s \\
\leq & \frac{\epsilon}{\Gamma(\alpha)} \int_{0}^{1}(1-s)^{\alpha-1} \sum_{i=1}^{4} a_{i}(s)\left(q_{i}+\epsilon\right) d s \\
& +\frac{\epsilon t}{\left|A_{\mu}\right| \Gamma(\alpha)} \int_{0}^{1}(1-s)^{\alpha-1} \sum_{i=1}^{4} a_{i}(s)\left(q_{i}+\epsilon\right) d s \\
& +\frac{\epsilon t}{\left|A_{\mu}\right| \Gamma(\alpha)} \int_{0}^{1}\left|H_{\alpha}(s)\right| \sum_{i=1}^{4} a_{i}(s)\left(q_{i}+\epsilon\right) d s .
\end{aligned}
$$


This implies that

$$
\begin{aligned}
\left|H_{\alpha}(s)\right| & \leq \int_{s}^{1}(z-s)^{\alpha-1}|h(z)| d z \leq \int_{s}^{1}(1-s)^{\alpha-1}|h(z)| d z \\
& \leq(1-s)^{\alpha-1} \int_{0}^{1}|h(z)| d z=(1-s)^{\alpha-1}\|h\|_{1} \leq(1-s)^{\alpha-2}\|h\|_{1}
\end{aligned}
$$

for all $s \in[0,1]$. Now for each $t \in[0,1]$ and $x, y \in X$ with $\|x-y\|_{*}<\frac{\delta}{\Delta}$, we have

$$
\begin{aligned}
\mid F_{x}(t) & -F_{y}(t) \mid \\
\leq & \frac{\epsilon}{\Gamma(\alpha)} \sum_{i=1}^{4}\left(q_{i}+\epsilon\right)\left[\int_{0}^{1}(1-s)^{\alpha-2} a_{i}(s) d s\right] \\
& +\frac{\epsilon t}{\left|A_{\mu}\right| \Gamma(\alpha)} \sum_{i=1}^{4}\left(q_{i}+\epsilon\right)\left[\int_{0}^{1}(1-s)^{\alpha-2} a_{i}(s) d s\right] \\
& +\frac{\epsilon t}{\left|A_{\mu}\right| \Gamma(\alpha)} \sum_{i=1}^{4}\left(q_{i}+\epsilon\right)\left[\int_{0}^{1}(1-s)^{\alpha-2} a_{i}(s) d s\right] \\
= & \left(\sum_{i=1}^{4}\left(q_{i}+\epsilon\right)\left\|\hat{a}_{i}\right\|_{[0,1]}\right)\left(\frac{1}{\Gamma(\alpha)}+\frac{t}{\left|A_{\mu}\right| \Gamma(\alpha)}+\frac{t\|h\|_{1}}{\left|A_{\mu}\right| \Gamma(\alpha)}\right) \epsilon .
\end{aligned}
$$

Thus,

$$
\left\|F_{x}-F_{y}\right\| \leq\left(\sum_{i=1}^{4}\left(q_{i}+\epsilon\right)\left\|\hat{a}_{i}\right\|_{[0,1]}\right)\left(\frac{1}{\Gamma(\alpha)}+\frac{1+\|h\|_{1}}{\left|A_{\mu}\right| \Gamma(\alpha)}\right) \epsilon
$$

for $\|x-y\|_{*}<\frac{\delta}{\Delta}$. Also for each $t \in[0,1]$ and $x, y \in X$, we have

$$
\begin{aligned}
\mid F_{x}^{\prime}(t) & -F_{y}^{\prime}(t) \mid \\
\leq & \frac{1}{\Gamma(\alpha-1)} \int_{0}^{t}(t-s)^{\alpha-2} \mid f\left(s, x(s), x^{\prime}(s), D^{\beta} x(s), \int_{0}^{s} g(\xi) x(\xi) d \xi\right) \\
& -f\left(s, y(s), y^{\prime}(s), D^{\beta} y(s), \int_{0}^{s} g(\xi) y(\xi) d \xi\right) \mid d s \\
& +\frac{1}{\left|A_{\mu}\right| \Gamma(\alpha)} \int_{0}^{\mu}(\mu-s)^{\alpha-1} \mid f\left(s, x(s), x^{\prime}(s), D^{\beta} x(s), \int_{0}^{s} g(\xi) x(\xi) d \xi\right) \\
& -f\left(s, y(s), y^{\prime}(s), D^{\beta} y(s), \int_{0}^{s} g(\xi) y(\xi) d \xi\right) \mid d s \\
& +\frac{1}{\left|A_{\mu}\right| \Gamma(\alpha)} \int_{0}^{1}\left|H_{\alpha}(s)\right| f\left(s, x(s), x^{\prime}(s), D^{\beta} x(s), \int_{0}^{s} g(\xi) x(\xi) d \xi\right) \\
& -f\left(s, y(s), y^{\prime}(s), D^{\beta} y(s), \int_{0}^{s} g(\xi) y(\xi) d \xi\right) \mid d s \\
\leq & \frac{1}{\Gamma(\alpha-1)} \int_{0}^{t}(t-s)^{\alpha-2}\left[a_{1}(s) \Lambda_{1}(|x(s)-y(s)|)+a_{2}(s) \Lambda_{2}\left(\left|x^{\prime}(s)-y^{\prime}(s)\right|\right)\right. \\
& \left.+a_{3}(s) \Lambda_{3}\left(\left|D^{\beta} x(s)-D^{\beta} y(s)\right|\right)+a_{4}(s) \Lambda_{4}\left(\left|\int_{0}^{s} g(\xi)(x(\xi)-y(\xi)) d \xi\right|\right)\right] d s
\end{aligned}
$$




$$
\begin{aligned}
& +\frac{1}{\left|A_{\mu}\right| \Gamma(\alpha)} \int_{0}^{\mu}(\mu-s)^{\alpha-1}\left[a_{1}(s) \Lambda_{1}(|x(s)-y(s)|)+a_{2}(s) \Lambda_{2}\left(\left|x^{\prime}(s)-y^{\prime}(s)\right|\right)\right. \\
& \left.+a_{3}(s) \Lambda_{3}\left(\left|D^{\beta} x(s)-D^{\beta} y(s)\right|\right)+a_{4}(s) \Lambda_{4}\left(\left|\int_{0}^{s} g(\xi)(x(\xi)-y(\xi)) d \xi\right|\right)\right] d s \\
& +\frac{1}{\left|A_{\mu}\right| \Gamma(\alpha)} \int_{0}^{1}\left|H_{\alpha}(s)\right|\left[a_{1}(s) \Lambda_{1}(|x(s)-y(s)|)+a_{2}(s) \Lambda_{2}\left(\left|x^{\prime}(s)-y^{\prime}(s)\right|\right)\right. \\
& \left.+a_{3}(s) \Lambda_{3}\left(\left|D^{\beta} x(s)-D^{\beta} y(s)\right|\right)+a_{4}(s) \Lambda_{4}\left(\left|\int_{0}^{s} g(\xi)(x(\xi)-y(\xi)) d \xi\right|\right)\right] d s \\
& \leq \frac{1}{\Gamma(\alpha-1)} \int_{0}^{t}(t-s)^{\alpha-2}\left[a_{1}(s) \Lambda_{1}(\|x-y\|)+a_{2}(s) \Lambda_{2}\left(\left\|x^{\prime}-y^{\prime}\right\|\right)\right. \\
& \left.\left.+a_{3}(s) \Lambda_{3}\left(\frac{\left\|x^{\prime}-y^{\prime}\right\|}{\Gamma(2-\beta)}\right)+a_{4}(s) \Lambda_{4}(m\|x-y\|)\right)\right] d s \\
& +\frac{1}{\left|A_{\mu}\right| \Gamma(\alpha)} \int_{0}^{\mu}(\mu-s)^{\alpha-1}\left[a_{1}(s) \Lambda_{1}(\|x-y\|)+a_{2}(s) \Lambda_{2}\left(\left\|x^{\prime}-y^{\prime}\right\|\right)\right. \\
& \left.+a_{3}(s) \Lambda_{3}\left(\frac{\left\|x^{\prime}-y^{\prime}\right\|}{\Gamma(2-\beta)}\right)+a_{4}(s) \Lambda_{4}(m\|x-y\|)\right] d s \\
& +\frac{1}{\left|A_{\mu}\right| \Gamma(\alpha)} \int_{0}^{1}\left|H_{\alpha}(s)\right|\left[a_{1}(s) \Lambda_{1}(\|x-y\|)+a_{2}(s) \Lambda_{2}\left(\left\|x^{\prime}-y^{\prime}\right\|\right)\right. \\
& \left.+a_{3}(s) \Lambda_{3}\left(\frac{\left\|x^{\prime}-y^{\prime}\right\|}{\Gamma(2-\beta)}\right)+a_{4}(s) \Lambda_{4}(m\|x-y\|)\right] d s \\
& \leq \frac{1}{\Gamma(\alpha-1)} \int_{0}^{t}(t-s)^{\alpha-2}\left[a_{1}(s) \Lambda_{1}\left(\Delta\|x-y\|_{*}\right)+a_{2}(s) \Lambda_{2}\left(\Delta\|x-y\|_{*}\right)\right. \\
& \left.+a_{3}(s) \Lambda_{3}\left(\Delta\|x-y\|_{*}\right)+a_{4}(s) \Lambda_{4}\left(\Delta\|x-y\|_{*}\right)\right] d s \\
& +\frac{1}{\left|A_{\mu}\right| \Gamma(\alpha)} \int_{0}^{\mu}(\mu-s)^{\alpha-1}\left[a_{1}(s) \Lambda_{1}\left(\Delta\|x-y\|_{*}\right)+a_{2}(s) \Lambda_{2}\left(\Delta\|x-y\|_{*}\right)\right. \\
& \left.+a_{3}(s) \Lambda_{3}\left(\Delta\|x-y\|_{*}\right)+a_{4}(s) \Lambda_{4}\left(\Delta\|x-y\|_{*}\right)\right] d s \\
& +\frac{1}{\left|A_{\mu}\right| \Gamma(\alpha)} \int_{0}^{1}\left|H_{\alpha}(s)\right|\left[a_{1}(s) \Lambda_{1}\left(\Delta\|x-y\|_{*}\right)+a_{2}(s) \Lambda_{2}\left(\Delta\|x-y\|_{*}\right)\right. \\
& \left.+a_{3}(s) \Lambda_{3}\left(\Delta\|x-y\|_{*}\right)+a_{4}(s) \Lambda_{4}\left(\Delta\|x-y\|_{*}\right)\right] d s .
\end{aligned}
$$

Thus, by using (5), we get

$$
\begin{aligned}
& \left|F_{x}^{\prime}(t)-F_{y}^{\prime}(t)\right| \\
& \leq \frac{\epsilon}{\Gamma(\alpha-1)} \int_{0}^{1}(1-s)^{\alpha-2} \sum_{i=1}^{4} a_{i}(s)\left(q_{i}+\epsilon\right) d s \\
& \quad+\frac{\epsilon}{\left|A_{\mu}\right| \Gamma(\alpha)} \int_{0}^{1}(1-s)^{\alpha-1} \sum_{i=1}^{4} a_{i}(s)\left(q_{i}+\epsilon\right) d s \\
& \quad+\frac{\epsilon}{\left|A_{\mu}\right| \Gamma(\alpha)} \int_{0}^{1}\left|H_{\alpha}(s)\right| \sum_{i=1}^{4} a_{i}(s)\left(q_{i}+\epsilon\right) d s
\end{aligned}
$$




$$
\begin{aligned}
\leq & \frac{\epsilon}{\Gamma(\alpha-1)} \sum_{i=1}^{4}\left(q_{i}+\epsilon\right)\left[\int_{0}^{1}(1-s)^{\alpha-2} a_{i}(s) d s\right] \\
& +\frac{\epsilon}{\left|A_{\mu}\right| \Gamma(\alpha)} \sum_{i=1}^{4}\left(q_{i}+\epsilon\right)\left[\int_{0}^{1}(1-s)^{\alpha-2} a_{i}(s) d s\right] \\
& +\frac{\epsilon}{\left|A_{\mu}\right| \Gamma(\alpha)} \sum_{i=1}^{4}\left(q_{i}+\epsilon\right)\left[\int_{0}^{1}(1-s)^{\alpha-2} a_{i}(s) d s\right] \\
= & \left(\sum_{i=1}^{4}\left(q_{i}+\epsilon\right)\left\|\hat{a}_{i}\right\|_{[0,1]}\right)\left(\frac{1}{\Gamma(\alpha)}+\frac{1}{\left|A_{\mu}\right| \Gamma(\alpha)}+\frac{\|h\|_{1}}{\left|A_{\mu}\right| \Gamma(\alpha)}\right) \epsilon
\end{aligned}
$$

whenever $\|x-y\|_{*}<\frac{\delta}{\Delta}$. Hence, $\left\|F_{x}^{\prime}-F_{y}^{\prime}\right\| \leq\left(\sum_{i=1}^{4}\left(q_{i}+\epsilon\right)\left\|\hat{a}_{i}\right\|_{[0,1]}\right)\left(\frac{1}{\Gamma(\alpha-1)}+\frac{1+\|h\|_{1}}{\left|A_{\mu}\right| \Gamma(\alpha)}\right) \epsilon$ whenever $\|x-y\|_{*}<\frac{\delta}{\Delta}$ and so

$$
\begin{aligned}
\left\|F_{x}-F_{y}\right\|_{*} & =\max \left\{\left\|F_{x}-F_{y}\right\|,\left\|F_{x}^{\prime}-F_{y}^{\prime}\right\|\right\} \\
& \leq\left(\sum_{i=1}^{4}\left(q_{i}+\epsilon\right)\left\|\hat{a}_{i}\right\|_{[0,1]}\right)\left(\frac{1}{\Gamma(\alpha-1)}+\frac{1+\|h\|_{1}}{\left|A_{\mu}\right| \Gamma(\alpha)}\right) \epsilon
\end{aligned}
$$

whenever $\|x-y\|_{*}<\frac{\delta}{\Delta}$. Since $\epsilon>0$ was arbitrary, $\left\|F_{x}-F_{y}\right\|_{*} \rightarrow 0$ whenever $\|x-y\|_{*} \rightarrow 0$. This implies that $F$ is continuous. Since $\lim _{z \rightarrow \infty} \frac{M_{i}(\Delta z, \ldots, \Delta z)}{\Delta z}=m_{i}$ for all $1 \leq i \leq k_{0}$, for each $\epsilon>0$ there exists $r(\epsilon)>0$ such that $\left|\frac{M_{i}(\Delta z, \ldots, \Delta z)}{\Delta z}-m_{i}\right|<\epsilon$ whenever $z \in[r(\epsilon), \infty)$. Thus,

$$
M_{i}(\Delta z, \ldots, \Delta z)<\left(m_{i}+\epsilon\right) \Delta z
$$

for all $z \in[r(\epsilon), \infty)$. Since $\lim _{z \rightarrow \infty} N(\Delta z, \ldots, \Delta z)<\infty, \lim _{z \rightarrow \infty} \frac{N(\Delta z, \ldots, \Delta z)}{\Delta z}=0$. Hence, there exists $r^{\prime}(\epsilon)>0$ such that $\frac{N(\Delta z, \ldots, \Delta z)}{\Delta z}<\epsilon$ and

$$
N(\Delta z, \ldots, \Delta z)<\Delta z \epsilon,
$$

for all $z \in\left[r^{\prime}(\epsilon), \infty\right)$. Since $\left(\frac{1}{\Gamma(\alpha-1)}+\frac{1+\|h\|_{1}}{\left|A_{\mu}\right| \Gamma(\alpha)}\right) \sum_{i=1}^{k_{0}} m_{i}\left\|\hat{\theta}_{i}\right\|_{[0,1]} \in\left(0, \frac{1}{\Delta}\right)$, there exists $\epsilon_{0}>0$ such that $\left(\frac{1}{\Gamma(\alpha-1)}+\frac{1+\|h\|_{1}}{\left|A_{\mu}\right| \Gamma(\alpha)}\right) \sum_{i=1}^{k_{0}}\left(m_{i}+\epsilon\right)\left\|\hat{\theta}_{i}\right\|_{[0,1]}+\left(\frac{1}{\Gamma(\alpha+1)}+\frac{\mu^{\alpha}+\|h\|_{1}}{\left|A_{\mu}\right| \Gamma(\alpha+1)}\right) \epsilon_{0} \in\left(0, \frac{1}{\Delta}\right)$. Now, put $r_{0}:=\max \left\{r\left(\epsilon_{0}\right), r^{\prime}\left(\epsilon_{0}\right)\right\}$. By using (7) and (8) for $z=r_{0}$, we get $M_{i}\left(\Delta r_{0}, \ldots, \Delta r_{0}\right)<\left(m_{i}+\epsilon_{0}\right) \Delta r_{0}$ and $N\left(\Delta r_{0}, \ldots, \Delta r_{0}\right)<\Delta r_{0} \epsilon_{0}$. Define $\Omega=\left\{x \in X:\|x\|_{*}<r_{0}\right\}$. Let $x_{0} \in \partial \Omega$ and $\lambda \in(0,1)$ be such that $x_{0}=\lambda F_{x_{0}}$. Then $\left\|x_{0}\right\|_{*}=r$. Now for each $t \in[0,1]$, we have

$$
x_{0}(t)=\lambda \int_{0}^{1} G(t, s) f\left(s, x(s), x^{\prime}(s), D^{\beta} x(s), \int_{0}^{s} g(\xi) x(\xi) d \xi\right) d s
$$

and so

$$
\begin{aligned}
& \left|x_{0}(t)\right| \\
& \quad=\left|\lambda \int_{0}^{1} G(t, s) f\left(s, x_{0}(s), x_{0}^{\prime}(s), D^{\beta} x_{0}(s), \int_{0}^{s} g(\xi) x_{0}(\xi) d \xi\right) d s\right| \\
& \quad \leq \lambda\left[\frac{1}{\Gamma(\alpha)} \int_{0}^{t}(t-s)^{\alpha-1} \sum_{i=1}^{k_{0}} \theta_{i}(s) M_{i}\left(x_{0}(s), x_{0}^{\prime}(s), D^{\beta} x_{0}(s), \int_{0}^{s} g(\xi) x_{0}(\xi) d \xi\right) d s\right.
\end{aligned}
$$


Talaee et al. Advances in Difference Equations

(2020) $2020: 41$

Page 11 of 16

$$
\begin{aligned}
& +\frac{1}{\Gamma(\alpha)} \int_{0}^{t}(t-s)^{\alpha-1} N\left(x_{0}(s), x_{0}^{\prime}(s), D^{\beta} x_{0}(s), \int_{0}^{s} g(\xi) x_{0}(\xi) d \xi\right) d s \\
& +\frac{t}{\left|A_{\mu}\right| \Gamma(\alpha)} \int_{0}^{\mu}(\mu-s)^{\alpha-1} \sum_{i=1}^{k_{0}} \theta_{i}(s) M_{i}\left(x_{0}(s), x_{0}^{\prime}(s), D^{\beta} x_{0}(s), \int_{0}^{s} g(\xi) x_{0}(\xi) d \xi\right) d s \\
& +\frac{t}{\left|A_{\mu}\right| \Gamma(\alpha)} \int_{0}^{\mu}(\mu-s)^{\alpha-1} N\left(x_{0}(s), x_{0}^{\prime}(s), D^{\beta} x_{0}(s), \int_{0}^{s} g(\xi) x_{0}(\xi) d \xi\right) d s \\
& +\frac{t}{\left|A_{\mu}\right| \Gamma(\alpha)} \int_{0}^{1} H_{\alpha}(s) \sum_{i=1}^{k_{0}} \theta_{i}(s) M_{i}\left(x_{0}(s), x_{0}^{\prime}(s), D^{\beta} x_{0}(s), \int_{0}^{s} g(\xi) x_{0}(\xi) d \xi\right) d s \\
& \left.+\frac{t}{\left|A_{\mu}\right| \Gamma(\alpha)} \int_{0}^{1} H_{\alpha}(s) \sum_{i=1}^{k_{0}} \theta_{i}(s) N\left(x_{0}(s), x_{0}^{\prime}(s), D^{\beta} x_{0}(s), \int_{0}^{s} g(\xi) x_{0}(\xi) d \xi\right) d s\right] \\
& \leq \lambda\left[\frac{1}{\Gamma(\alpha)} \sum_{i=1}^{k_{0}} \int_{0}^{t}(t-s)^{\alpha-1} \theta_{i}(s) M_{i}\left(\left\|x_{0}\right\|_{*},\left\|x_{0}\right\|_{*}, \frac{\left\|x_{0}\right\|_{*}}{\Gamma(2-\beta)}, m\left\|x_{0}\right\|_{*}\right) d s\right. \\
& +\frac{1}{\Gamma(\alpha)} \int_{0}^{t}(t-s)^{\alpha-1} N\left(\left\|x_{0}\right\|_{*},\left\|x_{0}\right\|_{*}, \frac{\left\|x_{0}\right\|_{*}}{\Gamma(2-\beta)}, m\left\|x_{0}\right\|_{*}\right) d s \\
& +\frac{t}{\left|A_{\mu}\right| \Gamma(\alpha)} \sum_{i=1}^{k_{0}} \int_{0}^{\mu}(\mu-s)^{\alpha-1} \theta_{i}(s) M_{i}\left(\left\|x_{0}\right\|_{*},\left\|x_{0}\right\|_{*}, \frac{\left\|x_{0}\right\|_{*}}{\Gamma(2-\beta)}, m\left\|x_{0}\right\|_{*}\right) d s \\
& +\frac{t}{\left|A_{\mu}\right| \Gamma(\alpha)} \int_{0}^{\mu}(\mu-s)^{\alpha-1} N\left(\left\|x_{0}\right\|_{*},\left\|x_{0}\right\|_{*}, \frac{\left\|x_{0}\right\|_{*}}{\Gamma(2-\beta)}, m\left\|x_{0}\right\|_{*}\right) d s \\
& +\frac{t}{\left|A_{\mu}\right| \Gamma(\alpha)} \sum_{i=1}^{k_{0}} \int_{0}^{1} H_{\alpha}(s) \theta_{i}(s) M_{i}\left(\left\|x_{0}\right\|_{*},\left\|x_{0}\right\|_{*}, \frac{\left\|x_{0}\right\|_{*}}{\Gamma(2-\beta)}, m\left\|x_{0}\right\|_{*}\right) d s \\
& \left.+\frac{t}{\left|A_{\mu}\right| \Gamma(\alpha)} \int_{0}^{1} H_{\alpha}(s) \sum_{i=1}^{k_{0}} \theta_{i}(s) N\left(\left\|x_{0}\right\|_{*},\left\|x_{0}\right\|_{*}, \frac{\left\|x_{0}\right\|_{*}}{\Gamma(2-\beta)}, m\left\|x_{0}\right\|_{*}\right) d s\right] \\
& \leq \lambda\left[\frac{1}{\Gamma(\alpha)} \sum_{i=1}^{k_{0}} M_{i}\left(\Delta r_{0}, \Delta r_{0}, \Delta r_{0}, \Delta r_{0}\right) \int_{0}^{1}(1-s)^{\alpha-2} \theta_{i}(s) d s\right. \\
& +\frac{1}{\Gamma(\alpha)} N\left(\Delta r_{0}, \Delta r_{0}, \Delta r_{0}, \Delta r_{0}\right) \int_{0}^{t}(t-s)^{\alpha-1} d s \\
& +\frac{t}{\left|A_{\mu}\right| \Gamma(\alpha)} \sum_{i=1}^{k_{0}} M_{i}\left(\Delta r_{0}, \Delta r_{0}, \Delta r_{0}, \Delta r_{0}\right) \int_{0}^{1}(1-s)^{\alpha-2} \theta_{i}(s) d s \\
& +\frac{t}{\left|A_{\mu}\right| \Gamma(\alpha)} N\left(\Delta r_{0}, \Delta r_{0}, \Delta r_{0}, \Delta r_{0}\right) \int_{0}^{\mu}(\mu-s)^{\alpha-1} d s \\
& +\frac{t}{\left|A_{\mu}\right| \Gamma(\alpha)}\|h\|_{1} \sum_{i=1}^{k_{0}} M_{i}\left(\Delta r_{0}, \Delta r_{0}, \Delta r_{0}, \Delta r_{0}\right) \int_{0}^{1}(1-s)^{\alpha-2} \theta_{i}(s) d s \\
& \left.+\frac{t}{\left|A_{\mu}\right| \Gamma(\alpha)}\|h\|_{1} N\left(\Delta r_{0}, \Delta r_{0}, \Delta r_{0}, \Delta r_{0}\right) \int_{0}^{1}(1-s)^{\alpha-1} d s\right] \\
& \leq \lambda\left[\frac{1}{\Gamma(\alpha)} \sum_{i=1}^{k_{0}} \Delta\left(m_{i}+\epsilon_{0}\right) r_{0}\left\|\hat{\theta}_{i}\right\|_{[0,1]}+\frac{t^{\alpha}}{\Gamma(\alpha+1)} \Delta r_{0} \epsilon_{0}\right.
\end{aligned}
$$




$$
\begin{aligned}
& +\frac{t}{\left|A_{\mu}\right| \Gamma(\alpha)} \sum_{i=1}^{k_{0}} \Delta\left(m_{i}+\epsilon_{0}\right) r_{0}\left\|\hat{\theta}_{i}\right\|_{[0,1]}+\frac{t \mu^{\alpha}}{\left|A_{\mu}\right| \Gamma(\alpha+1)} \Delta r_{0} \epsilon_{0} \\
& \left.+\frac{t\|h\|_{1}}{\left|A_{\mu}\right| \Gamma(\alpha)} \sum_{i=1}^{k_{0}} \Delta\left(m_{i}+\epsilon_{0}\right) r_{0}\left\|\hat{\theta}_{i}\right\|_{[0,1]}+\frac{t\|h\|_{1}}{\left|A_{\mu}\right| \Gamma(\alpha+1)} \Delta r_{0} \epsilon_{0}\right]
\end{aligned}
$$

for all $t \in[0,1]$. Hence,

$$
\begin{aligned}
\left\|x_{0}\right\| \leq & \lambda\left[\left(\frac{1}{\Gamma(\alpha)}+\frac{1+\|h\|_{1}}{\left|A_{\mu}\right| \Gamma(\alpha)}\right) \sum_{i=1}^{k_{0}}\left(m_{i}+\epsilon_{0}\right)\left\|\hat{\theta}_{i}\right\|_{[0,1]}\right. \\
& \left.+\left(\frac{1}{\Gamma(\alpha+1)}+\frac{\mu^{\alpha}+\|h\|_{1}}{\left|A_{\mu}\right| \Gamma(\alpha+1)}\right) \epsilon_{0}\right] \Delta r_{0} \\
\leq & \lambda\left[\left(\frac{1}{\Gamma(\alpha-1)}+\frac{1+\|h\|_{1}}{\left|A_{\mu}\right| \Gamma(\alpha)}\right) \sum_{i=1}^{k_{0}}\left(m_{i}+\epsilon_{0}\right)\left\|\hat{\theta}_{i}\right\|_{[0,1]}\right. \\
& \left.+\left(\frac{1}{\Gamma(\alpha+1)}+\frac{\mu^{\alpha}+\|h\|_{1}}{\left|A_{\mu}\right| \Gamma(\alpha+1)}\right) \epsilon_{0}\right] \Delta r_{0} \\
< & r_{0} .
\end{aligned}
$$

Also for each $t \in[0,1]$, we have

$$
\begin{aligned}
\left|x_{0}^{\prime}(t)\right| & \left|\lambda \int_{0}^{1} \frac{\partial G}{\partial t}(t, s) f\left(s, x_{0}(s), x_{0}^{\prime}(s), D^{\beta} x_{0}(s), \int_{0}^{s} g(\xi) x_{0}(\xi) d \xi\right) d s\right| \\
\leq & \lambda\left[\frac{1}{\Gamma(\alpha-1)} \int_{0}^{t}(t-s)^{\alpha-2} \sum_{i=1}^{k_{0}} \theta_{i}(s) M_{i}\left(x_{0}(s), x_{0}^{\prime}(s), D^{\beta} x_{0}(s), \int_{0}^{s} g(\xi) x_{0}(\xi) d \xi\right) d s\right. \\
& +\frac{1}{\Gamma(\alpha-1)} \int_{0}^{t}(t-s)^{\alpha-2} N\left(x_{0}(s), x_{0}^{\prime}(s), D^{\beta} x_{0}(s), \int_{0}^{s} g(\xi) x_{0}(\xi) d \xi\right) d s \\
& +\frac{1}{\left|A_{\mu}\right| \Gamma(\alpha)} \int_{0}^{\mu}(\mu-s)^{\alpha-1} \sum_{i=1}^{k_{0}} \theta_{i}(s) M_{i}\left(x_{0}(s), x_{0}^{\prime}(s), D^{\beta} x_{0}(s), \int_{0}^{s} g(\xi) x_{0}(\xi) d \xi\right) d s \\
& +\frac{1}{\left|A_{\mu}\right| \Gamma(\alpha)} \int_{0}^{\mu}(\mu-s)^{\alpha-1} N\left(x_{0}(s), x_{0}^{\prime}(s), D^{\beta} x_{0}(s), \int_{0}^{s} g(\xi) x_{0}(\xi) d \xi\right) d s \\
& +\frac{1}{\left|A_{\mu}\right| \Gamma(\alpha)} \int_{0}^{1} H_{\alpha}(s) \sum_{i=1}^{k_{0}} \theta_{i}(s) M_{i}\left(x_{0}(s), x_{0}^{\prime}(s), D^{\beta} x_{0}(s), \int_{0}^{s} g(\xi) x_{0}(\xi) d \xi\right) d s \\
& \left.+\frac{1}{\left|A_{\mu}\right| \Gamma(\alpha)} \int_{0}^{1} H_{\alpha}(s) \sum_{i=1}^{k_{0}} \theta_{i}(s) N\left(x_{0}(s), x_{0}^{\prime}(s), D^{\beta} x_{0}(s), \int_{0}^{s} g(\xi) x_{0}(\xi) d \xi\right) d s\right] \\
\leq & \lambda\left[\frac{1}{\Gamma(\alpha-1)} \sum_{i=1}^{k_{0}} \int_{0}^{t}(t-s)^{\alpha-2} \theta_{i}(s) M_{i}\left(\left\|x_{0}\right\|_{*},\left\|x_{0}\right\|_{*}, \frac{\left\|x_{0}\right\|_{*}}{\Gamma(2-\beta)}, m\left\|x_{0}\right\|_{*}\right) d s\right. \\
& +\frac{1}{\Gamma(\alpha-1)} \int_{0}^{t}(t-s)^{\alpha-2} N\left(\left\|x_{0}\right\|_{*},\left\|x_{0}\right\|_{*}, \frac{\left\|x_{0}\right\|_{*}}{\Gamma(2-\beta)}, m\left\|x_{0}\right\|_{*}\right) d s
\end{aligned}
$$




$$
\begin{aligned}
& +\frac{1}{\left|A_{\mu}\right| \Gamma(\alpha)} \sum_{i=1}^{k_{0}} \int_{0}^{\mu}(\mu-s)^{\alpha-1} \theta_{i}(s) M_{i}\left(\left\|x_{0}\right\|_{*},\left\|x_{0}\right\|_{*}, \frac{\left\|x_{0}\right\|_{*}}{\Gamma(2-\beta)}, m\left\|x_{0}\right\|_{*}\right) d s \\
& +\frac{1}{\left|A_{\mu}\right| \Gamma(\alpha)} \int_{0}^{\mu}(\mu-s)^{\alpha-1} N\left(\left\|x_{0}\right\|_{*},\left\|x_{0}\right\|_{*}, \frac{\left\|x_{0}\right\|_{*}}{\Gamma(2-\beta)}, m\left\|x_{0}\right\|_{*}\right) d s \\
& +\frac{1}{\left|A_{\mu}\right| \Gamma(\alpha)} \sum_{i=1}^{k_{0}} \int_{0}^{1} H_{\alpha}(s) \theta_{i}(s) M_{i}\left(\left\|x_{0}\right\|_{*},\left\|x_{0}\right\|_{*}, \frac{\left\|x_{0}\right\|_{*}}{\Gamma(2-\beta)}, m\left\|x_{0}\right\|_{*}\right) d s \\
& \left.+\frac{1}{\left|A_{\mu}\right| \Gamma(\alpha)} \int_{0}^{1} H_{\alpha}(s) \sum_{i=1}^{k_{0}} \theta_{i}(s) N\left(\left\|x_{0}\right\|_{*},\left\|x_{0}\right\|_{*}, \frac{\left\|x_{0}\right\|_{*}}{\Gamma(2-\beta)}, m\left\|x_{0}\right\|_{*}\right) d s\right] \\
& \leq \lambda\left[\frac{1}{\Gamma(\alpha-1)} \sum_{i=1}^{k_{0}} M_{i}\left(\Delta r_{0}, \Delta r_{0}, \Delta r_{0}, \Delta r_{0}\right) \int_{0}^{1}(1-s)^{\alpha-2} \theta_{i}(s) d s\right. \\
& +\frac{1}{\Gamma(\alpha-1)} N\left(\Delta r_{0}, \Delta r_{0}, \Delta r_{0}, \Delta r_{0}\right) \int_{0}^{t}(t-s)^{\alpha-2} d s \\
& +\frac{1}{\left|A_{\mu}\right| \Gamma(\alpha)} \sum_{i=1}^{k_{0}} M_{i}\left(\Delta r_{0}, \Delta r_{0}, \Delta r_{0}, \Delta r_{0}\right) \int_{0}^{1}(1-s)^{\alpha-2} \theta_{i}(s) d s \\
& +\frac{1}{\left|A_{\mu}\right| \Gamma(\alpha)} N\left(\Delta r_{0}, \Delta r_{0}, \Delta r_{0}, \Delta r_{0}\right) \int_{0}^{\mu}(\mu-s)^{\alpha-1} d s \\
& +\frac{1}{\left|A_{\mu}\right| \Gamma(\alpha)}\|h\|_{1} \sum_{i=1}^{k_{0}} M_{i}\left(\Delta r_{0}, \Delta r_{0}, \Delta r_{0}, \Delta r_{0}\right) \int_{0}^{1}(1-s)^{\alpha-2} \theta_{i}(s) d s \\
& \left.+\frac{1}{\left|A_{\mu}\right| \Gamma(\alpha)}\|h\|_{1} N\left(\Delta r_{0}, \Delta r_{0}, \Delta r_{0}, \Delta r_{0}\right) \int_{0}^{1}(1-s)^{\alpha-1} d s\right] \\
& \leq \lambda\left[\frac{1}{\Gamma(\alpha-1)} \sum_{i=1}^{k_{0}} \Delta\left(m_{i}+\epsilon_{0}\right) r_{0}\left\|\hat{\theta}_{i}\right\|_{[0,1]}+\frac{t^{\alpha-1}}{\Gamma(\alpha)} \Delta r_{0} \epsilon_{0}\right. \\
& +\frac{1}{\left|A_{\mu}\right| \Gamma(\alpha)} \sum_{i=1}^{k_{0}} \Delta\left(m_{i}+\epsilon_{0}\right) r_{0}\left\|\hat{\theta}_{i}\right\|_{[0,1]}+\frac{\mu^{\alpha}}{\left|A_{\mu}\right| \Gamma(\alpha+1)} \Delta r_{0} \epsilon_{0} \\
& \left.+\frac{\|h\|_{1}}{\left|A_{\mu}\right| \Gamma(\alpha)} \sum_{i=1}^{k_{0}} \Delta\left(m_{i}+\epsilon_{0}\right) r_{0}\left\|\hat{\theta}_{i}\right\|_{[0,1]}+\frac{\|h\|_{1}}{\left|A_{\mu}\right| \Gamma(\alpha+1)} \Delta r_{0} \epsilon_{0}\right] .
\end{aligned}
$$

Thus, $\left\|x_{0}^{\prime}\right\| \leq \lambda\left[\left(\frac{1}{\Gamma(\alpha-1)}+\frac{1+\|h\|_{1}}{\left|A_{\mu}\right| \Gamma(\alpha)}\right) \sum_{i=1}^{k_{0}}\left(m_{i}+\epsilon_{0}\right)\left\|\hat{\theta}_{i}\right\|_{[0,1]}+\left(\frac{1}{\Gamma(\alpha+1)}+\frac{\mu^{\alpha}+\|h\|_{1}}{\left|A_{\mu}\right| \Gamma(\alpha+1)}\right) \epsilon_{0}\right] \Delta r_{0}<r_{0}$ and so $\left\|x_{0}\right\|_{*}=\max \left\{\left\|x_{0}\right\|,\left\|x_{0}^{\prime}\right\|\right\}<r_{0}$. Now by using Lemma $2, F$ has a fixed point in $\bar{\Omega}$ and so the problem (1) has a solution.

\section{Example 1 Consider the problem}

$$
\begin{aligned}
& D^{\frac{5}{2}} x(t)+\theta(t) M\left(x(t), x^{\prime}(t), D^{\frac{1}{2}} x(t), \int_{0}^{t} \xi x(\xi) d \xi\right)+N\left(x(t), x^{\prime}(t), D^{\frac{1}{2}} x(t), \int_{0}^{t} \xi x(\xi) d \xi\right) \\
& \quad=0
\end{aligned}
$$

with boundary conditions $x\left(\frac{2}{3}\right)=\int_{0}^{1} z x(z) d z$ and $x(0)=x^{\prime \prime}(0)=0$, where $M\left(x_{1}, \ldots, x_{4}\right)=$ $\Sigma_{i=1}^{4}\left|x_{i}\right|, N\left(x_{1}, \ldots, x_{4}\right)=\sum_{i=1}^{4} \frac{\left|x_{i}\right|}{1+\left|x_{i}\right|}, \theta(t)=\frac{1}{50 \sqrt{1-t}(t)}$ and $p(t)=0$ whenever $t \in[0,1] \cap \mathcal{Q}$ 
and $p(t)=1$ as $t \in[0,1] \cap \mathcal{Q}^{c}$. Put $f\left(t,\left(x_{1}, \ldots, x_{4}\right)=\theta(t) M\left(x_{1}, \ldots, x_{4}\right)+N\left(x_{1}, \ldots, x_{4}\right), k_{0}=\right.$ $1, M_{1}\left(x_{1}, \ldots, x_{4}\right):=M\left(x_{1}, \ldots, x_{4}\right), \theta_{1}(t):=\theta(t), a_{1}(t)=\cdots=a_{4}(t):=1+\theta(t), \Lambda_{1}(z)=\cdots=$ $\Lambda_{4}(z)=z, g_{1}(z)=\cdots=g_{4}(z)=g(t)=h(t)=t, \alpha=\frac{5}{2}, \beta=\frac{1}{2}, \lambda=\frac{1}{2}$ and $\mu=\frac{2}{3}$. Then we have

$$
\begin{aligned}
& \left|f\left(t, x_{1}, x_{2}, \ldots, x_{4}\right)-f\left(t, y_{1}, y_{2}, \ldots, y_{4}\right)\right| \\
& \quad=\theta(t)\left|\sum_{i=1}^{4}\right| x_{i}|-| y_{i}||+\left|\sum_{i=1}^{4} \frac{\left(1+\left|y_{i}\right|\right)\left|x_{i}\right|-\left(1+\left|x_{i}\right|\right)\left|y_{i}\right|}{\left(1+\left|x_{i}\right|\right)\left(1+\left|y_{i}\right|\right)}\right| \\
& \quad \leq \theta(t) \sum_{i=1}^{4}\left|x_{i}-y_{i}\right|+\sum_{i=1}^{4} \frac{\left|x_{i}-y_{i}\right|}{\left(1+\left|x_{i}\right|\right)\left(1+\left|y_{i}\right|\right)} \\
& \quad \leq \theta(t) \sum_{i=1}^{4}\left|x_{i}-y_{i}\right|+\sum_{i=1}^{4}\left|x_{i}-y_{i}\right| \\
& \quad=(\theta(t)+1) \sum_{i=1}^{4}\left|x_{i}-y_{i}\right|=\sum_{i=1}^{4} a_{i}(t) \Lambda_{i}\left(\left|x_{i}-y_{i}\right|\right) .
\end{aligned}
$$

One can see that $\hat{a}_{i}(t)=(1-s)^{\alpha-2} a_{i}(t) \in L^{1}[0,1], \Lambda_{i}: \mathbb{R}^{+} \rightarrow \mathbb{R}^{+}$is nondecreasing, $\lim _{z \rightarrow 0^{+}} \frac{\Lambda_{i}(z)}{g_{i}(z)}=1:=q_{i} \in[0, \infty)$ and $\lim _{z \rightarrow 0^{+}} g_{i}(z)=0$ for $1 \leq i \leq 4$. Also, $M$ and $N$ are nondecreasing with respect to all their components, $\lim _{z \rightarrow \infty} \frac{M_{1}(z, \ldots, z)}{z}=4:=m_{1} \in(0, \infty)$ and $\lim _{z \rightarrow \infty} N(z, \ldots, z)=4<\infty$. Note that, $g, h \in L^{1}[0,1], \int_{0}^{1} z h(z) d z=\frac{1}{3} \neq \mu, m:=\|g\|_{1}=\frac{1}{2}$, $A_{\mu}=\int_{0}^{1}(z h(z)-\mu) d z=\int_{0}^{1}\left(z^{2}-\frac{2}{3}\right) d z=\frac{1}{3}, \Delta:=\max \left\{1, m, \frac{1}{\Gamma(2-\beta)}\right\}=\frac{2}{\sqrt{\pi}}$ and $\left\|\hat{\theta_{1}}\right\|_{[0,1]}=\frac{1}{50}$.

Finally, we have

$$
\begin{aligned}
& \left(\frac{1}{\Gamma(\alpha-1)}+\frac{1+\|h\|_{1}}{\left|A_{\mu}\right| \Gamma(\alpha)}\right) \sum_{i=1}^{k_{0}} m_{i}\left\|\hat{\theta}_{i}\right\|_{[0,1]} \\
& \quad=\left(\frac{1}{\Gamma\left(\frac{3}{2}\right)}+\frac{1+\frac{1}{2}}{\frac{1}{3} \Gamma\left(\frac{5}{2}\right)}\right) \times 4 \times \frac{1}{50} \\
& \quad=\left(\frac{2}{\sqrt{\pi}}+6 \sqrt{\pi}\right) \times 4 \times \frac{1}{50} \in\left(0, \frac{2}{\sqrt{\pi}}\right)=\left(0, \frac{1}{\Delta}\right) .
\end{aligned}
$$

Thus by using Theorem 4, this problem has a solution.

\section{Conclusion}

It is important that we increase our ability for studying of complicate fractional integrodifferential equation. One of such equations are pointwise defined multi-singular fractional differential equations. Solving of such equations prepares us for modeling of most phenomena without removing most parameters which play a role in the phenomena. It is natural that most software is not able to calculate solutions of most singular differential equations now, while this weakliness relates to the structures of the softwares. In this work, we study the existence of solutions for a pointwise defined multi-singular fractional differential equation under some integral boundary conditions.

\section{Acknowledgements}

The first and third authors were supported by South Tehran Branch, Islamic Azad University. The fourth author was supported by Azarbaijan Shahid Madani University. The authors express their gratitude to the dear unknown referees for their helpful suggestions which improved the final version of this paper. 
Funding

Not available.

\section{Availability of data and materials}

Data sharing not applicable to this article as no datasets were generated or analyzed during the current study.

\section{Ethics approval and consent to participate}

Not applicable.

\section{Competing interests}

The authors declare that they have no competing interests.

\section{Consent for publication}

Not applicable.

\section{Authors' contributions}

The authors declare that the study was realized in collaboration with equal responsibilities. All authors read and approved the final manuscript.

\section{Author details}

'Department of Mathematics, South Tehran Branch, Islamic Azad University, Tehran, Iran. ${ }^{2}$ Department of Mathematics, Mehran Branch, Islamic Azad University, Mehran, Iran. ${ }^{3}$ Department of Mathematics, Azarbaijan Shahid Madani University, Tabriz, Iran. ${ }^{4}$ Department of Medical Research, China Medical University Hospital, China Medical University, Taichung, Taiwan.

\section{Publisher's Note}

Springer Nature remains neutral with regard to jurisdictional claims in published maps and institutional affiliations.

Received: 24 November 2019 Accepted: 13 January 2020 Published online: 20 January 2020

\section{References}

1. Ahmad, B., Etemad, S., Ettefagh, M., Rezapour, S.: On the existence of solutions for fractional $q$-difference inclusions with q-antiperiodic boundary conditions. Bull. Math. Soc. Sci. Math. Roum. 59(2), 119-134 (2016)

2. Baleanu, D., Ghafarnezhad, K., Rezapour, S.: On a three steps crisis integro-differential equation. Adv. Differ. Equ. 2018, 135 (2018). https://doi.org/10.1186/s13662-019-2088-2

3. Baleanu, D., Mohammadi, H., Rezapour, S.: On a nonlinear fractional differential equation on partially ordered metric spaces. Adv. Differ. Equ. 2013, 83 (2013). https://doi.org/10.1186/1687-1847-2013-83

4. Baleanu, D., Rezapour, S., Mohammadi, H.: Some existence results on nonlinear fractional differential equations. Philos. Trans. R. Soc. A, Math. Phys. Eng. Sci. 371, 7 (2013). https://doi.org/10.1098/rsta.2012.0144

5. Baleanu, D., Mohammadi, H., Rezapour, S.: The existence of solutions for a nonlinear mixed problem of singular fractional differential equations. Adv. Differ. Equ. 2013, 359 (2013). https://doi.org/10.1186/1687-1847-2013-359

6. Baleanu, D., Hedayati, V., Rezapour, S., Al-Qurashi, M.M.: On two fractional differential inclusions. SpringerPlus 5(1), 882 (2016)

7. Baleanu, D., Khan, H., Jafari, H., Khan, R.A., Alipour, M.: On existence results for solutions of a coupled system of hybrid boundary value problems with hybrid conditions. Adv. Differ. Equ. 2015, 318 (2015)

8. Baleanu, D., Rezapour, S., Etemad, S., Alsaedi, A.: On a time-fractional integro-differential equation via three-point boundary value conditions. Math. Probl. Eng. 2015, 12 (2015)

9. Bragdi, M., Debbouche, A., Baleanu, D.: Existence of solutions for fractional differential inclusions with separated boundary conditions in Banach space. Adv. Math. Phys. 2013, 5 (2013)

10. Hristova, S., Tunc, C.: Stability of nonlinear Volterra integro-differential equations with Caputo fractional derivative and bounded delays. Electron. J. Differ. Equ. 2019(30), 11 (2019)

11. Khan, H., Tunc, C., Chen, W., Khan, A.: Existence theorems and Hyers-Ulam stability for a class of hybrid fractional differential equations with p-Laplacian operator. J. Appl. Anal. Comput. 8(4), 1211-1226 (2018)

12. Ntouyas, S.K., Etemad, S.: On the existence of solutions for fractional differential inclusions with sum and integral boundary conditions. Appl. Math. Comput. 266, 235-243 (2015)

13. Samei, M.E., Hedayati, V., Rezapour, S.: Existence results for a fraction hybrid differential inclusion with Caputo-Hadamard type fractional derivative. Adv. Differ. Equ. 2019, 163 (2019). https://doi.org/10.1186/s13662-019-2090-8

14. Aydogan, M.S., Baleanu, D., Mousalou, A., Rezapour, S.: On high order fractional integro-differential equations including the Caputo-Fabrizio derivative. Bound. Value Probl. 2018(1), 90 (2018). https://doi.org/10.1186/s13661-018-1008-9

15. Aydogan, S.M., Baleanu, D., Mousalou, A., Rezapour, S.: On approximate solutions for two higher-order Caputo-Fabrizio fractional integro-differential equations. Adv. Differ. Equ. 2017(1), 221 (2017). https://doi.org/10.1186/s13662-017-1258-3

16. Baleanu, D., Mousalou, A., Rezapour, S.: A new method for investigating approximate solutions of some fractional integro-differential equations involving the Caputo-Fabrizio derivative. Adv. Differ. Equ. 2017(1), 51 (2017). https://doi.org/10.1186/s13662-017-1088-3

17. Baleanu, D., Mousalou, A., Rezapour, S.: The extended fractional Caputo-Fabrizio derivative of order $0 \leq \sigma<1$ on $C_{\mathbb{P}}[0,1]$ and the existence of solutions for two higher-order series-type differential equations. Adv. Differ. Equ. 2018(1), 255 (2018). https://doi.org/10.1186/s13662-018-1696-6 
18. Baleanu, D., Mousalou, A., Rezapour, S.: On the existence of solutions for some infinite coefficient-symmetric Caputo-Fabrizio fractional integro-differential equations. Bound. Value Probl. 2017(1), 145 (2017). https://doi.org/10.1186/s13661-017-0867-9

19. Baleanu, D., Rezapour, S., Saberpour, Z.: On fractional integro-differential inclusions via the extended fractional Caputo-Fabrizio derivation. Bound. Value Probl. 2019, 79 (2019). https://doi.org/10.1186/s13661-019-1194-0

20. Baleanu, D., Jajarmi, A., Sajjadi, S.S., Mozyrska, D.: A new fractional model and optimal control of a tumor-immune surveillance with non-singular derivative operator. Chaos 29, 083127 (2019). https://doi.org/10.1063/1.5096159

21. Hajipour, M., Jajarmi, A., Baleanu, D.: On the accurate discretization of a highly nonlinear boundary value problem. Numer. Algorithms 79, 679-695 (2018)

22. Hajipour, M., Jajarmi, A., Malek, A., Baleanu, D.: Positivity-preserving sixth-order implicit finite difference weighted essentially non-oscillatory scheme for the nonlinear heat equation. Appl. Math. Comput. 325, 146-158 (2018)

23. Jajarmi, A., Arshad, S., Baleanu, D.: A new fractional modelling and control strategy for the outbreak of dengue fever. Phys. A, Stat. Mech. Appl. 535, 122524 (2019). https://doi.org/10.1016/.jphysa.2019.122524

24. Jajarmi, A., Baleanu, D., Sajjadi, S.S., Asad, J.H.: A new feature of the fractional Euler-Lagrange equations for a coupled oscillator using a nonsingular operator approach. Front. Phys. 7, 196 (2019)

25. Jajarmi, A., Ghanbari, B., Baleanu, D.: A new and efficient numerical method for the fractional modeling and optimal control of diabetes and tuberculosis co-existence. Chaos 29, 093111 (2019). https://doi.org/10.1063/1.5112177

26. Goswami, A., Singh, J., Kumar, D., Gupta, S., Sushila: An efficient analytical technique for fractional partial differential equations occurring in ion acoustic waves in plasma. J. Ocean Eng. Sci. 4(2), 85-99 (2019)

27. Goswami, A., Singh, J., Kumar, D., Gupta, S., Sushila: An efficient analytical approach for fractional equal width equations describing hydro-magnetic waves in cold plasma. Phys. A, Stat. Mech. Appl. 524, 563-575 (2019)

28. Kumar, D., Singh, J., Baleanu, D.: On the analysis of vibration equation involving a fractional derivative with Mittag-Leffler law. Math. Methods Appl. Sci. 43(1), 443-457 (2020)

29. Singh, J., Kumar, D., Baleanu, D.: New aspects of fractional Biswas-Milovic model with Mittag-Leffler law. Math. Model. Nat. Phenom. 14(3), 303 (2019)

30. Singh, J., Kumar, D., Baleanu, D., Rathore, S.: On the local fractional wave equation in fractal strings. Math. Methods Appl. Sci. 42(5), 1588-1595 (2019)

31. Mohammadi, A., Aghazadeh, N., Rezapour, S.: Haar wavelet collocation method for solving singular and nonlinear fractional time-dependent Emden-Fowler equations with initial and boundary conditions. Math. Sci. 13, 255-265 (2019)

32. Liu, Y., Wong, P.J.Y.: Global existence of solutions for a system of singular fractional differential equations with impulse effects. J. Appl. Math. Inform. 33(3-4), 327-342 (2015)

33. Shabibi, M., Postolache, M., Rezapour, S., Vaezpour, S.M.: Investigation of a multisingular pointwise defined fractional integro-differential equation. J. Math. Anal. 7(5), 61-77 (2016)

34. Baleanu, D., Ghafarnezhad, K., Rezapour, S., Shabibi, M.: On the existence of solutions of a three steps crisis integro-differential equation. Adv. Differ. Equ. 2018, 135 (2018). https://doi.org/10.1186/s13662-018-1583-1

35. Chergui, D., Oussaeif, T.E., Ahcene, M.: Existence and uniqueness of solutions for nonlinear fractional differential equations depending on lower-order derivative with non-separated type integral boundary conditions. AIMS Math. 4(1), 112-133 (2019)

36. Podlubny, I.: Fractional Differential Equations. Academic Press, San Diego (1999)

37. Samko, S.G., Kilbas, A.A., Marichev, O.I.: Fractional Integrals and Derivatives: Theory and Applications. Gordon and Breach Science Publishers, Switzerland (1993)

38. Zeidler, E:: Nonlinear Functional Analysis and Its Applications-l: Fixed Point Theorems. Springer, USA (1986)

\section{Submit your manuscript to a SpringerOpen ${ }^{\circ}$ journal and benefit from:}

- Convenient online submission

- Rigorous peer review

- Open access: articles freely available online

- High visibility within the field

- Retaining the copyright to your article

Submit your next manuscript at $\gg$ springeropen.com 